\begin{tabular}{|c|l|}
\hline Title & $\begin{array}{l}\text { A nalysis of magnetic random telegraph noise in individual arrangements of a small number of coupled MnA s } \\
\text { nanoclusters }\end{array}$ \\
\hline Author(s) & Fischer, Martin; Elm, Matthias T.; Kato, Hiroaki; Sakita, Shinya; Hara, Shinjiro; Klar, Peter J. \\
\hline Citation & $\begin{array}{l}\text { Physical Review B, 92(16), 165306 } \\
\text { https://doi.org/10.1103/PhysRevB.92.165306 }\end{array}$ \\
\hline Issue Date & 2015-10-06 \\
\hline Doc URL & http://hdl.handle.net/2115/59889 \\
\hline Rights & @2015A merican Physical Society \\
\hline Type & article \\
\hline File Information & PhysRevB.92.165306.pdf \\
\hline
\end{tabular}

Instructions for use 


\title{
Analysis of magnetic random telegraph noise in individual arrangements of a small number of coupled MnAs nanoclusters
}

\author{
Martin Fischer, ${ }^{1}$ Matthias T. Elm,,${ }^{1,2, *}$ Hiroaki Kato, ${ }^{3}$ Shinya Sakita, ${ }^{3}$ Shinjiro Hara, ${ }^{3}$ and Peter J. Klar ${ }^{1}$ \\ ${ }^{1}$ Institute of Experimental Physics I, Justus-Liebig University, Heinrich-Buff-Ring 16, 35392 Giessen, Germany \\ ${ }^{2}$ Institute of Physical Chemistry, Justus-Liebig University, Heinrich-Buff-Ring 58, 35392 Giessen, Germany \\ ${ }^{3}$ Research Center for Integrated Quantum Electronics, Hokkaido University, North 13, West 8, Sapporo 060-8628, Japan
}

(Received 11 June 2015; revised manuscript received 20 August 2015; published 6 October 2015)

\begin{abstract}
The temporal dependence of the resistance of MnAs nanocluster arrangements grown by selective-area metal-organic vapor-phase epitaxy is investigated at different temperatures. The resistance of such arrangements exhibits random telegraph noise with jumps between discrete resistance levels. The effect is attributed to thermally activated switching of the magnetic domain structure resulting in alterations of spin-dependent scattering between the MnAs clusters of the arrangements. The behavior can be qualitatively understood by a simple model in which it is assumed that the nanocluster arrangement consists of three domains in accordance with investigations by magnetic force microscopy. The magnetizations of the outer larger domains remain fixed, whereas the magnetization of a smaller intermediate domain (or domain wall) exhibits thermally activated switching between local minima of its energy landscape. The results of the model indicate that the time scale of an actual switching event of the entire intermediate domain comprises the nucleation of a seed domain consisting of a few thousand Mn spins followed by the transformation of the entire domain by domain-wall motion in order to reorient its magnetization.
\end{abstract}

DOI: 10.1103/PhysRevB.92.165306

\section{INTRODUCTION}

New concepts in magnetoelectronics for nonvolatile, highdensity memory $[1,2]$ and logic devices $[3,4]$ as well as the improvement of magnetic sensors [5] require the miniaturization of magnetic elements to the submicron scale. A fundamental limit for miniaturization of magnetic building blocks is the critical size below which thermally activated fluctuations of the magnetization play a dominant role [6]. Thus, a detailed understanding of the thermally activated switching behavior of the magnetization in magnetic single-domain particles, of its size dependence, and of the underlying energy landscape is essential for optimizing magnetoelectronic devices for future applications. In recent years intensive research on magnetization reversal as well as magnetic noise in microand nanoscale systems as a function of temperature and external magnetic field has been performed [7-9]. At lower frequencies miniaturized magnetic systems such as singledomain particles [10-14], magnetic disks [15], nanowires [16], and microscale magnetic tunnel junctions [17] often show magnetic random telegraph noise (RTN), where the resistance fluctuates between two discrete resistance values. These resistance fluctuations are attributed to thermally activated changes of the magnetic structure, such as random switching of the magnetization between minima in the energy landscape of the system, making the analysis of magnetic RTN a powerful tool for investigating the magnetic properties $[10,11]$.

Ferromagnetic MnAs is a promising material system for spin electronics [18] and magnetoelectronic applications $[19,20]$. Growth of high-quality MnAs nanoclusters by selective-area metal-organic vapor-phase epitaxy (SA-MOVPE) offers a number of advantages in the design of lateral magnetoelectronic device structures as degrees of freedom such as size, shape, and position can easily be

*matthias.elm@exp1.physik.uni-giessen.de
PACS number(s): 73.50.Td, 73.63.Bd, 75.75.-c, 81.16.-c tuned and controlled [21-23]. Recently, a spin-valve-like behavior was reported for an arrangement of two MnAs nanoclusters $[20,24]$. In such devices the thermal influence on the magnetic structure of MnAs nanocluster arrangements is rather unexplored so far, although thermally activated magnetic switching phenomena may have a severe impact on the device performance $[20,24]$.

In this paper we report on magnetic RTN of high-quality, single-crystalline MnAs nanocluster arrangements, which represent a suitable model system for studying magnetization dynamics in nanoscale systems as their magnetic properties such as anisotropy constants are well known.

\section{EXPERIMENTAL RESULTS}

The nanocluster arrangements were grown by SA-MOVPE on prepatterned (111)B-GaAs substrates covered with a growth-inhibiting $\mathrm{SiO}_{2}$ mask layer. Thus, the cluster growth only took place in mask openings intentionally prepared, making it possible to actively tune the clusters' size, shape, and position [25-28]. Figure 1 shows scanning electron microscopy images of the two nanocluster arrangements prepared. The first arrangement [Fig. 1(a)] consists of one single, lateral nanowire with a length of about $3 \mu \mathrm{m}$ and a width of about $450 \mathrm{~nm}$. It was grown in a corresponding single wirelike mask opening. The second arrangement shown in Fig. 1(b) consists of two elongated nanoclusters, which have a length of approximately 1000 and $550 \mathrm{~nm}$ and width of about 320 and $220 \mathrm{~nm}$, respectively. Two separate mask openings were defined in the $\mathrm{SiO}_{2}$, and the two elongated clusters merged during the MOVPE growth process, forming a larger bent cluster with its two legs aligned at an angle of $120^{\circ}$. Electrical contacts were prepared on both nanocluster arrangements using electron-beam lithography followed by thermal evaporation of $\mathrm{Ti}(10 \mathrm{~nm})$ and $\mathrm{Au}(100 \mathrm{~nm})$.

Time-dependent resistance measurements were performed at different temperatures. Corresponding resistance traces are 
(a)

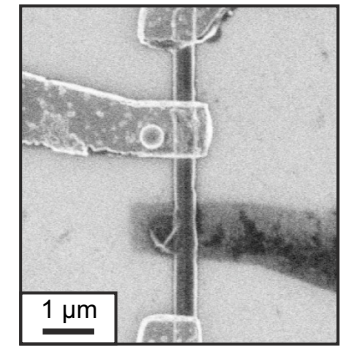

(c)

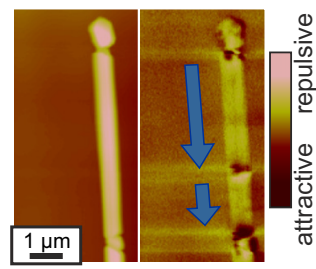

(b)

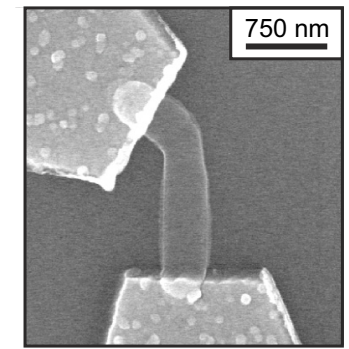

(d)

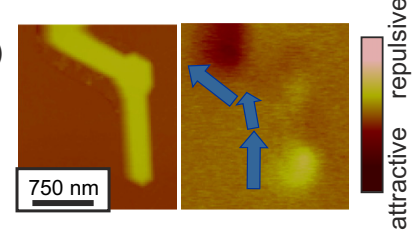

FIG. 1. (Color online) (a) Scanning electron microscopy images of the lateral MnAs nanowire and (b) the bent nanocluster arrangement investigated. Atomic force microscopy and MFM images of a comparable (c) lateral nanowire (taken from Ref. [28]) and (d) bent nanocluster showing the magnetic domain structures of the arrangements.

shown in Fig. 2. Clear signatures of RTN, i.e., abrupt changes in the resistance between well-defined resistance levels, can be detected for both arrangements. As the frequency of the resistance jumps depends exponentially on temperature, RTN could only be investigated in a sample-specific narrow temperature range with a width of about $15-20 \mathrm{~K}$. At lower temperatures no resistance jumps were observed as the switching times became too long to yield good statistics of the switching events, whereas at higher temperatures the frequency of the resistance jumps was too high to resolve the discrete resistance levels with the measurement setup used. While only two resistance levels occur for the lateral MnAs nanowire, as shown in Fig. 2(a), the resistance of the bent nanocluster fluctuates between three discrete levels [Fig. 2(b)]. As mentioned above, RTN may arise due to thermal activated switching of the magnetization of single magnetic domains in the nanoclusters (magnetic RTN) but is also often observed in metal oxide semiconductors or metal-insulator-metal junctions, where it originates from trapping and untrapping of electrons by a defect at a barrier or interface [29,30]. While nonmagnetic RTN is independent of an external magnetic field [10,11,17,31], magnetic RTN can be suppressed by a strong magnetic field as it destroys the domain structure and aligns the entire magnetization of the sample. In order to exclude trapping of electrons at defects at the nanocluster-metal interface as the source of the RTN observed, time-dependent measurements were performed at different external magnetic fields. As shown in Fig. 2(c), the RTN of the bent nanocluster strongly depends on the magnetic field applied and completely vanishes at fields above $3 \mathrm{~T}$, confirming that magnetic RTN is observed. It is worth noting that the bent nanocluster shows four resistance levels at $110 \mathrm{~K}$ and $\mu_{0} H=0 \mathrm{~T}$. As will be discussed later, this behavior is attributed to changes in the magnetic structure of the nanocluster at higher temperatures. The fluctuating magnetic domain is only stable in a narrow temperature range,
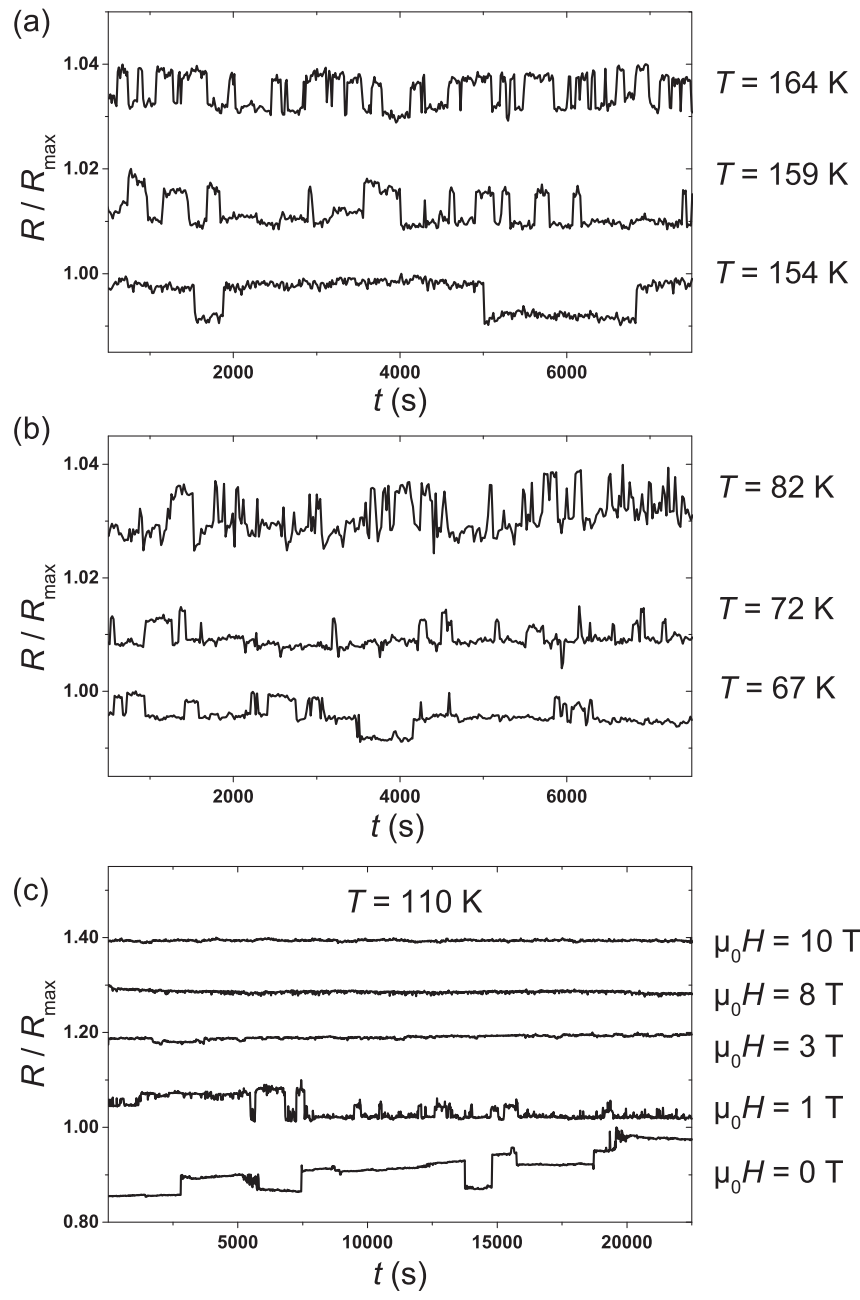

FIG. 2. (a) Random telegraph noise of the single MnAs nanowire and (b) of the bent nanocluster arrangement determined at different temperatures. (c) Suppression of the RTN of the bent nanocluster by an external magnetic field.

which additionally limits the accessible temperature range for the analysis of the magnetic RTN.

The formation of magnetic domains in lateral MnAs nanowires was investigated in detail by Kato et al. [28] using magnetic force microscopy (MFM). They found that lateral nanowires can exhibit a complex domain structure depending on the nanocluster's dimensions [28]. Figures 1(c) and 1(d) show MFM images of noncontacted clusters similar to the ones studied in the electrical measurements, showing the typical domain structure of such clusters. Both arrangements exhibit two large domains whose magnetizations are oriented parallel to the elongation directions of the nanoclusters. For the bent nanocluster [Fig. 1(d)] a third, small magnetic domain is visible in the region where the elongated clusters merge. As described by Heiliger et al., the conductivity of an ideal two-domain system (without domain-wall structure but with ideal interfaces) depends on the relative angle $\phi$ between the orientations of the magnetizations and possesses a cosine-like dependence on $\phi$ [32]. For a parallel alignment of the two magnetizations the spin-dependent scattering at the ideal interface is minimal, resulting in a low resistance, while for an 
antiparallel alignment the spin-dependent scattering and thus the resistance are maximal.

\section{MODELLING AND ANALYSIS OF THE MAGNETIZATION FLUCTUATIONS}

Based on the MFM results, we propose a simple model which explains the resistance fluctuations of the nanocluster arrangements on a semiquantitative level. In the model, it is assumed that the RTN arises from thermally activated fluctuations of the magnetization of a small domain (or fluctuations of the magnetic structure of a domain wall) located between two larger domains whose magnetizations are fixed for simplicity.

As described by Néel and Brown [34,36], the switching frequency $f$ of the magnetization of a small ferromagnetic single-domain particle exhibits an Arrhenius behavior:

$$
f=\frac{1}{\tau}=f_{0} \exp \left(-\frac{E_{\mathrm{B}}}{k_{\mathrm{B}} T}\right),
$$

where $k_{\mathrm{B}}$ denotes the Boltzmann constant, $f_{0}$ is the attempt frequency, $\tau$ is the average residence time of the magnetization in a particular orientation state (i.e., in a particular local minimum of the energy landscape of the magnetization), and $E_{\mathrm{B}}$ denotes the energy barrier height between two minima. Thus, by analyzing the fluctuations as a function of temperature, the energy barrier height in the energy landscape may be estimated. For the fluctuating, intermediate domain of the MnAs nanoclusters investigated here the magnetization should be oriented in the sample plane, as MnAs nanoclusters grown on (111)B-GaAs substrates exhibit a hard magnetic axis along the $c$ direction [21], i.e., perpendicular to the sample surface. Thus, the free energy $U_{m}$ of the magnetization $m=|\vec{m}|$ of the intermediate domain with volume $V_{m}$ as a function of its in-plane magnetization angle $\varphi_{m}$ can be written as

$$
\begin{aligned}
U_{m}\left(\varphi_{m}\right)= & -J_{1} m M_{1} \cos \left(\varphi_{m}-\Phi_{1}\right)-J_{2} m M_{2} \cos \left(\varphi_{m}-\Phi_{2}\right) \\
& +K_{\text {Cryst }, m} V_{m} \cos \left(6 \varphi_{m}\right) \\
& +K_{\text {Shape }, m} V_{m} \sin ^{2}\left(\varphi_{m}-\varepsilon_{m}\right),
\end{aligned}
$$

where $M_{1}=\left|\vec{M}_{1}\right|$ and $M_{2}=\left|\vec{M}_{2}\right|$ are the magnetizations of the larger outer domains, assumed to be thermally stable and oriented at a fixed angle of $\Phi_{1}$ and $\Phi_{2}$, respectively. The first two terms with coupling constants $J_{1}$ and $J_{2}$ describe the coupling of the magnetization $\vec{m}$ to $\vec{M}_{1}$ and $\vec{M}_{2}$. The third term with the anisotropy constant $K_{\mathrm{Cryst}, m}$ represents the effect of the sixfold symmetry of the magnetocrystalline anisotropy of MnAs on $\vec{m}[21,33,35]$, and the fourth term accounts for a shape anisotropy of the intermediate magnetic domain with an anisotropy constant $K_{\text {Shape, } m}$ and an orientation along $\varepsilon_{m}$. The free energy $U_{m}$ basically describes the energy landscape in which $\vec{m}$ fluctuates.

In order to determine the energy barriers of the fluctuating domain in the MnAs nanocluster arrangements, the magnetic RTN signals were analyzed to extract the mean residence time $\bar{\tau}$ of each resistance level. Figure 3(a) shows the temperature dependence of the mean residence times $\bar{\tau}_{\mathrm{H}}$ and $\bar{\tau}_{\mathrm{L}}$ determined for the high and low resistance levels of the lateral MnAs nanowire. Both resistance levels exhibit almost the same Arrhenius-type behavior of $\bar{\tau}$ and yield comparable energy

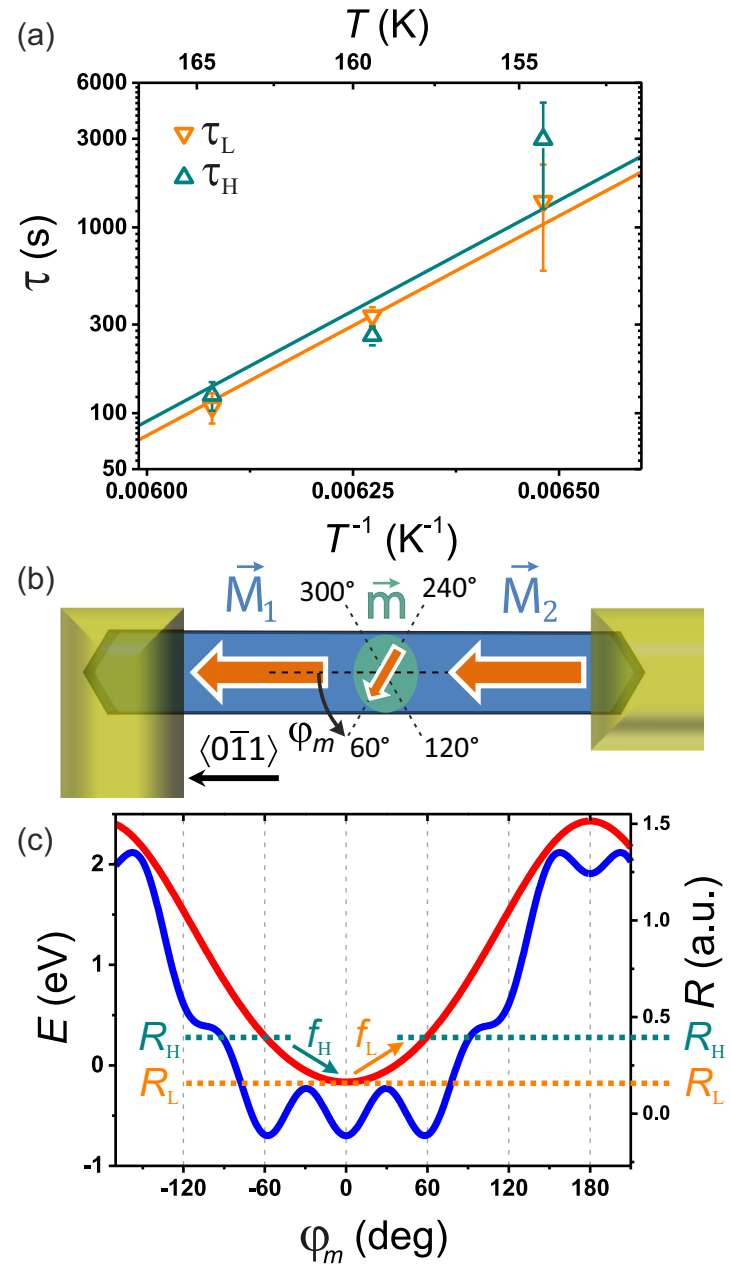

FIG. 3. (Color online) (a) Arrhenius plot of the mean residence times of the two resistance levels observed for the lateral MnAs nanowire. Solid lines represent fits to the experimental data. (b) Illustration of the three-domain model explaining the observed two-level resistance fluctuations. (c) Proposed energy landscape of the intermediate domain and corresponding resistance of the cluster arrangement.

barriers $E_{\mathrm{B}}$. The magnetic structure of this lateral nanowire can be described according to the MFM data given above by two thermally stable domains oriented parallel to the elongated direction of the nanowire and one small intermediate domain (or domain wall), whose magnetization fluctuates between minima of the energy landscape described according to Eq. (2). The domain structure assumed is schematically shown in Fig. 3(b).

If one took into account solely the magnetocrystalline anisotropy and neglected effects due to shape anisotropy and coupling of $\vec{m}$ to $\vec{M}_{1}$ and $\vec{M}_{2}$, one would obtain an energy landscape for $\vec{m}$ with six equivalent minima located at angles $\varphi_{m}=0^{\circ}, \pm 60^{\circ}, \pm 120^{\circ}$, and $180^{\circ}$, respectively. Presuming a cosine-like dependence of the interface resistance between adjacent domains as described by Heiliger et al. [32], the six minima would result in four different resistance levels. The resistance values of the arrangement for $\varphi= \pm 60^{\circ}$ would be degenerate; the same would hold for those at $\varphi= \pm 120^{\circ}$. However, the observation of two resistance levels only in the RTN measurements of this sample suggests that the effects 
of shape anisotropy and coupling between the magnetizations are required to obtain a more likely energy landscape. The Néel-type domain structure usually observed in elongated MnAs nanowires [37] can be described by a shape anisotropy of the intermediate domain perpendicular to the nanowire's magnetization orientation, i.e., $\varepsilon_{m}=90^{\circ}$. Taking this shape anisotropy and the coupling between the clusters' magnetizations into account yields the energy landscape depicted in Fig. 3(c). This energy landscape exhibits now only four minima, three minima at about $\varphi=0^{\circ}$ and $\pm 60^{\circ}$, where $\vec{m}$ somewhat points in the same direction as $\vec{M}_{1}$ and $\vec{M}_{2}$, and one for an antiparallel orientation of $\vec{m}$, i.e., $\varphi_{m}=180^{\circ}$. These would correspond to three resistance levels. However, as the energy difference, which needs to be overcome to reach the minima at $\varphi=180^{\circ}$, i.e., antiparallel alignment of $\vec{m}$ with respect to $\vec{M}_{1}$ and $\vec{M}_{2}$, is about $3 \mathrm{eV}$, the probability that $\vec{m}$ resides in this antiparallel orientation is negligible. Therefore, as shown in Fig. 3(c), only two resistance levels remain, denoted by $R_{\mathrm{H}}$ and $R_{\mathrm{L}}$ in accordance with the experimental results. The former corresponds to the degenerate orientations described by $\varphi= \pm 60^{\circ}$, and the latter corresponds to $\varphi=0^{\circ}$. Usually, two-level telegraph noise is modeled by a two-well system separated by one energy barrier. This model can also be applied to the lateral nanowire arrangement as the energy landscape is symmetric with respect to $\varphi=0^{\circ}$. Changes in the mean residence time of the high-resistance state due to its degeneracy are compensated by having two possibilities to switch for the magnetization residing at $\varphi=0^{\circ}$.

For a more quantitative description, Eq. (1) was used to calculate the transition rates between the energy minima. For simplicity, only jumps between adjacent minima were considered. The mean residence time $\bar{\tau}_{\mathrm{H}}$ is therefore given by the transition of the magnetization from the minima at $\varphi_{m}= \pm 60^{\circ}$ to $\varphi_{m}=0^{\circ}$ and $\bar{\tau}_{\mathrm{L}}$ by the opposite switching of the magnetization. The corresponding energy barriers $E_{\mathrm{B}, \mathrm{H}}$ and $E_{\mathrm{B}, \mathrm{L}}$ are determined by the energy landscape of the intermediate domain. To fit the calculated mean residence times to the experimental data, the coupling constants $J_{1}$ and $J_{2}$, the attempt frequency $f_{0}$, and the volume $V_{m}$ of the intermediate domain were used as fitting parameters. The magnetization of each domain was calculated assuming a magnetic moment per Mn atom [38,39] of $3.4 \mu_{\mathrm{B}}$ and a Mn particle density [40] of $n_{\mathrm{Mn}}=2.96 \times 10^{28} \mathrm{~m}^{-3}$, i.e., $M_{1}=M_{2}=m=3.4 \mu_{\mathrm{B}} n_{\mathrm{Mn}}=$ $9.33 \times 10^{5} \mathrm{~J} / \mathrm{Tm}^{3}$. The shape anisotropy constant [41] can then be calculated yielding $K_{\text {Shape } m}=\frac{\mu_{0}}{2} M^{2}=5.46 \times 10^{5} \mathrm{~J} / \mathrm{m}^{3}$. For the magnetocrystalline anisotropy constant a value of $K_{\text {Cryst }, m}=-1.62 \times 10^{5} \mathrm{~J} / \mathrm{m}^{3}$ was used, which was determined previously by ferromagnetic resonance measurements of hexagonal-shaped MnAs nanoclusters [42]. A satisfactory agreement between the calculations and the experimental results was obtained for the fitting parameters listed in Table I; the corresponding fitted curve is shown in Fig. 3(a) as a solid line. A value of $f_{0}=1 \times 10^{12} \mathrm{~Hz}$ was obtained for the attempt frequency, in good agreement with attempt frequencies derived from theory for single-domain particles $[43,44]$ and comparable to values determined for permalloy dots [10] or superparamagnetic particles $[45,46]$, which were in the range from $10^{9}$ to $10^{13} \mathrm{~Hz}$. For both coupling constants a value of about $(1.2 \pm 0.1) \times 10^{-31} \mathrm{~J} / \mathrm{Tm}^{3}$ is obtained. For the volume of
TABLE I. Attempt frequencies and fitting parameters of the energy landscape for both nanocluster arrangements.

\begin{tabular}{|c|c|c|c|c|c|}
\hline Arrangement & level & $\begin{array}{c}f_{0} \\
(\mathrm{~Hz})\end{array}$ & $\begin{array}{c}J_{1} \\
\left(\mathrm{~J} / \mathrm{Tm}^{3}\right)\end{array}$ & $\begin{array}{c}J_{2} \\
\left(\mathrm{~J} / \mathrm{Tm}^{3}\right)\end{array}$ & $\begin{array}{c}V_{m} \\
\left(\mathrm{~nm}^{3}\right)\end{array}$ \\
\hline Nanowire & $\mathrm{H}, \mathrm{L}$ & $1 \times 10^{12}$ & $1.2 \times 10^{-31}$ & $1.2 \times 10^{-31}$ & 250 \\
\hline Nanocluster & $\begin{array}{c}\mathrm{H} \\
\mathrm{I} \\
\mathrm{L}\end{array}$ & $\begin{array}{c}0.07 \\
1.6 \\
70\end{array}$ & $1.9 \times 10^{-32}$ & $0.5 \times 10^{-32}$ & 40 \\
\hline
\end{tabular}

the fluctuating domain a value of $V_{m}=(2.5 \pm 0.1) \times 10^{-25} \mathrm{~m}^{3}$ is derived; that is, approximately $7400 \mathrm{Mn}$ atoms are involved in the coherent rotation process of the magnetization, which is comparable to fluctuating magnetization volumes responsible for magnetic RTN in other magnetic systems [11,47]. The small value for $V_{m}$ indicates that the magnetic RTN arises from thermally activated changes of only a small fraction of the domain wall between the two large magnetic domains.

Using the parameters obtained, the energy barriers $E_{\mathrm{B}, \mathrm{H}}$ and $E_{\mathrm{B}, \mathrm{L}}$ were calculated, yielding values of 0.469 and $0.472 \mathrm{eV}$, respectively. These values are in reasonably good agreement with those determined from a linear fit to the data points in Fig. 3(a), $E_{\mathrm{B}, \mathrm{H}}=0.388 \pm 0.17 \mathrm{eV}$ and $E_{\mathrm{B}, \mathrm{L}}=$ $0.509 \pm 0.023 \mathrm{eV}$. The comparatively large error bars are due to the restricted temperature range, where RTN could be observed. In a two-level system, where detailed balance is obeyed, the energy difference $\Delta E$ between the two states can be determined from the ratio of the mean residence times [12]:

$$
\frac{\bar{\tau}_{\mathrm{H}}}{\bar{\tau}_{\mathrm{L}}}=\frac{P_{\mathrm{H}}}{P_{\mathrm{L}}}=\exp \left(\frac{\Delta E}{k_{\mathrm{B}} T}\right),
$$

where $P_{\mathrm{H}}$ and $P_{\mathrm{L}}$ are the probabilities to be in the high- and low-resistance states, respectively. Using Eq. (3) an energy difference of about $(3 \pm 6) \mathrm{meV}$ is obtained, which agrees well with the energy difference determined from the energy landscape. Thus, within the experimental error a detailed balance is obeyed for the lateral MnAs nanowire.

The same analysis was also performed for the mean residence times of the bent nanocluster. The temperature dependence of the extracted residence times is shown in an Arrhenius representation in Fig. 4(a). Here $\bar{\tau}_{\mathrm{H}}, \bar{\tau}_{\mathrm{I}}$, and $\bar{\tau}_{\mathrm{L}}$ denote the mean residence times of the highest, the intermediate, and the lowest resistance levels, respectively. In contrast to the results obtained for the lateral nanowire, the temperature dependence of the mean residence times of the bent cluster exhibits different activation energies, indicating a more complex energy landscape. In accordance with the MFM investigations [Fig. 1(d)] a three-domain structure with two large, thermally stable domains parallel to $\Phi_{1}=0^{\circ}$ and $\Phi_{2}=$ $60^{\circ}$ and a third smaller intermediate domain was assumed, as illustrated in Fig. 4(b). Good agreement between the model and experiment was achieved by assuming a shape anisotropy of the intermediate domain with its long axis at an angle $\varepsilon_{m}=$ $110^{\circ}$ and coupling between adjacent domains in addition to the magnetocrystalline anisotropy. The temperature dependence for the three residence times $\bar{\tau}_{\mathrm{H}}, \bar{\tau}_{\mathrm{I}}$, and $\bar{\tau}_{\mathrm{L}}$ derived on the basis of the modeling is plotted as solid lines in Fig. 4(a). The fitting 
(a)

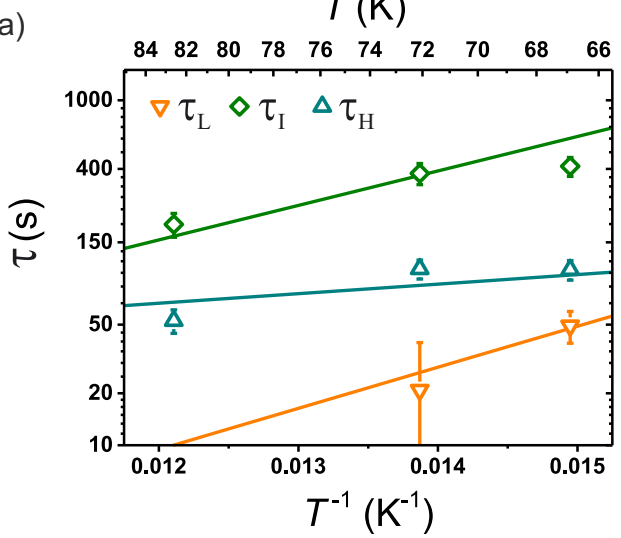

(b)
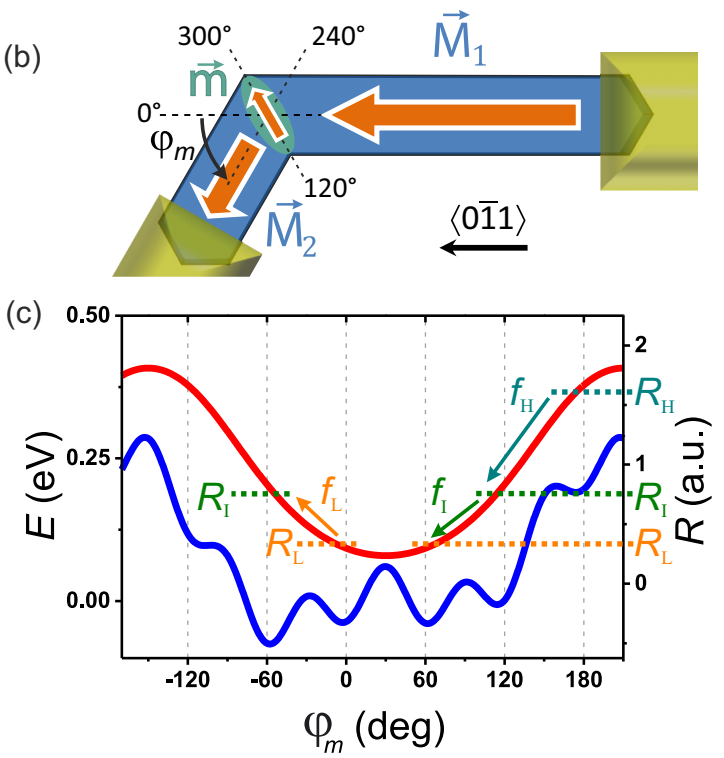

FIG. 4. (Color online) (a) Arrhenius plot of the mean residence times of the three resistance levels observed for the bent nanocluster arrangement. Solid lines represent fits to the experimental data. (b) Illustration of the three-domain model explaining the observed three-level resistance fluctuations. (c) Proposed energy landscape of the intermediate domain and corresponding resistance of the cluster arrangement.

parameters yielding the best agreement are listed in Table I. The corresponding energy landscape and the resistance as a function of $\varphi_{m}$ are plotted in Fig. 4(c). The energy landscape reveals five magnetization orientations, which corresponds to three different resistance values. It exhibits a maximum barrier height of less than $0.3 \mathrm{eV}$; that is, thermally activated switching between all five magnetization orientations has to be considered. Again, only magnetization switching between adjacent minima, which leads to a change in the resistance, was considered in order to derive the mean residence times; that is, the jumps of $\vec{m}$ from $\varphi_{m}=0^{\circ}$ to $60^{\circ}$ and vice versa were neglected, as the resistances corresponding to the two orientations are the same and thus cannot be distinguished experimentally. As a consequence, direct jumps between a low-resistance state and the high-resistance state at $\varphi=180^{\circ}$ are also excluded, which is in agreement with the experimental results, where no such jumps are observed. Due to the existence of three resistance levels, the behavior of the mean residence times cannot be described by a two-level system as in the case of the lateral MnAs nanowire. For example, $\bar{\tau}_{\mathrm{I}}$ is given by the mean residence time of the state at $\varphi=-60^{\circ}$ and $120^{\circ}$, which can switch to three states at $\varphi=0^{\circ}, 60^{\circ}$, and $180^{\circ}$; that is, $\bar{\tau}_{\mathrm{I}}$ is determined by two different states and three different energy barriers. Furthermore, as will be discussed below, the attempt frequencies differ for the three different resistance levels. Thus, an analysis of the mean residence times using Eq. (3) is not applicable.

The energy barriers were determined from the temperature dependence of the mean residence times as described above, yielding values of $68.7 \pm 8,23.5 \pm 7$, and $20.9 \pm 10 \mathrm{meV}$ for the low, intermediate, and high resistance levels, respectively. Calculating the energy barriers from the proposed energy landscape, one obtains values of $E_{\mathrm{B}, \mathrm{L}}=72.8 \mathrm{meV}, E_{\mathrm{B}, \mathrm{I}}=$ $39.6 \mathrm{meV}$, and $E_{\mathrm{B}, \mathrm{H}}=11 \mathrm{meV}$ for the lowest energy barrier of each resistance state, which is in an acceptable agreement with the barrier heights obtained from the linear fit. The fitted magnetization volume $V_{m}=(4 \pm 1) \times 10^{-26} \mathrm{~m}^{3}$ of the intermediate domain of the bent arrangement is much smaller than that of the lateral MnAs nanowire. It corresponds to only 1200 simultaneously switching Mn spins. The two coupling constants $J_{1}=(1.9 \pm 0.1) \times 10^{-32} \mathrm{~J} / \mathrm{Tm}^{3}$ and $J_{2}=$ $(0.5 \pm 0.1) \times 10^{-32} \mathrm{~J} / \mathrm{Tm}^{3}$ differ for this arrangement. This can be understood when considering that the coupling constant as defined in Eq. (2) includes the volumes of both coupled magnetizations. The volume of single magnetic domain 1 oriented along $\Phi_{1}=0^{\circ}$ is a factor of about 2.6 larger than the volume of domain 2. The ratio of their volumes of 2.6 is comparable to the corresponding ratio of $3.8 \pm 0.9$ of their coupling constants.

Although the proposed energy landscape of the bent nanocluster arrangement yields a good description of the temperature dependence of each $\bar{\tau}$, which is determined by only the energy barriers, a satisfactory agreement between the fitted curve and the experimental mean residence times could only be achieved by assuming three different attempt frequencies, i.e., one for each resistance level. The values of these attempt frequencies range from $70 \mathrm{~Hz}$ for the high resistance level to $0.07 \mathrm{~Hz}$ for the low resistance level and are several orders of magnitude smaller than attempt frequencies of $10^{9}$ to $10^{13} \mathrm{~Hz}$ predicted for the switching of single magnetic particles as discussed above. However, as the attempt frequency depends on several additional parameters, e.g., damping, gyromagnetic ratio, and temperature [48], the range of experimentally observed values is much broader and covers values from $10^{3}$ to $10^{13} \mathrm{~Hz}[11,49,50]$. Furthermore, the attempt frequency also strongly depends on the shape of the particle. Bode et al. investigated the attempt frequency of superparamagnetic Fe nanoislands and determined values strongly scattering between $2 \times 10^{-8}$ and $5 \times 10^{11} \mathrm{~Hz}$ [14]. An increased switching rate was found for elongated islands compared to compact ones, which was attributed to domainwall formation. Magnetic reversal processes induced by seed nucleation and propagation of domain walls led to an effective increase of the attempt frequencies. A similar effect was also reported for nanosized $\mathrm{Ni}$ wires [47] and submicron-sized magnetic thin films [7]. In such a case, the effective attempt frequency $f_{0}$ not only is determined by the time for coherent magnetization reversal of the seed but also includes the time 
for the remainder of the magnetic domain to reorient its spins. Such a situation may also occur for the MnAs nanocluster arrangements studied here, where the switching volumes $V_{m}$ $\left(\approx 6.3\right.$ and $3.5 \mathrm{~nm}^{3}$, respectively) are significantly smaller than the actual volume assigned to the intermediate domain (or domain wall) according to the MFM measurements. Thus, $V_{m}$ can be interpreted as the volume of the coherently switching seed, whose newly established domain wall then propagates through the intermediate domain and fully realigns its magnetization. A rough estimate of the switching time can be obtained from the actual resistance jumps themselves, where the transition between two resistance levels requires a period of time of something like 5 to $10 \mathrm{~s}$. If one assumes that this time interval corresponds to the time required for the propagation of the new spin orientation (defined by the seed nucleus) across the entire length of the intermediate domain in order to fully switch its magnetization, one may estimate the domain-wall velocity. The intermediate region of the bent nanocluster arrangement has a width of about $300 \mathrm{~nm}$, yielding a domain-wall velocity of 30 to $60 \mathrm{~nm} / \mathrm{s}$. This velocity value is comparable to velocities of thermally driven domain-wall motion observed in $\mathrm{Co} / \mathrm{Pt}$ multilayer nanowires [51]. The different attempt frequencies observed for the two types of nanocluster arrangements may then be attributed to differences in the domain-wall character or the propagation path through the cluster arrangement. But pinning at defects and geometrical pinning sites, which strongly influence the dynamic behavior of magnetic domains [52-54], or the formation of more than one single magnetic domain $[16,55]$ may also be responsible for the increased attempt frequencies extracted by our simple model based only on three domains. In particular, when considering the shape of the bent nanocluster with two large magnetic domains oriented in a relative angle of $60^{\circ}$, the formation of a more complex domain structure in the intermediate region going beyond the simple model may be anticipated.

Investigations of the magnetic field behavior of the RTN usually offer the possibility to study the domain structure in more detail [10-12,17]. In the case of the bent nanocluster arrangement, magnetic-field-dependent measurements of the RTN were performed at $110 \mathrm{~K}$, as shown in Fig. 2(c). At this temperature four resistance levels are observed, indicating a more complex domain structure with more than one fluctuating domain (or domain wall); that is, the domain structure is thermally stable only in a certain narrow temperature range. Thus, a detailed analysis of the magnetic field dependence of the RTN was not possible. Nevertheless, the influence of the magnetic field on the RTN can be explained qualitatively when assuming an energy landscape for the fluctuating magnetic domains as described in Fig. 4(c). Applying a magnetic field of $\mu_{0} H=1 \mathrm{~T}$ parallel to $M_{1}$, i.e., along $\varphi=0^{\circ}$, increases the mean residence time of the low-resistance state as the energy of the state at $\varphi=0^{\circ}$ decreases. Furthermore, the adjacent energy barriers are reduced, resulting in an increased switching rate between the low and intermediate resistance levels in accordance with the experimental observations. At higher magnetic fields, the energy landscape exhibits only one minimum parallel to the magnetic field direction, resulting in the suppression of the magnetic RTN.

\section{SUMMARY AND CONCLUSIONS}

In summary, we reported on magnetic RTN of arrangements consisting of single-crystalline MnAs nanoclusters grown by SA-MOVPE. The results obtained demonstrate that due to their well-known magnetic properties these MnAs nanoclusters are a suitable model system to investigate thermally activated changes of the magnetic structure in nanoscale systems. The single crystallinity of the nanoclusters makes them superior to magnetic nanostructures prepared by top-down processes, where surface or structural defects may affect the magnetization switching behavior. Furthermore, the possibility to tune the clusters' size, shape, and orientation with respect to each other offers the possibility to study the influence of the systems' dimensions on the magnetization dynamics systematically.

\section{ACKNOWLEDGMENTS}

The authors gratefully acknowledge financial support from the DFG. This work was also financially supported in part by the Japan-Germany Research Cooperative Program from the JSPS and DAAD.
[1] G. A. Prinz, Science 282, 1660 (1998).

[2] S. S. P. Parkin, M. Hayashi, and L. Thomas, Science 320, 190 (2008).

[3] R. P. Cowburn and M. E. Welland, Science 287, 1466 (2000).

[4] D. A. Allwood, G. Xiong, C. C. Faulkner, D. Atkinson, D. Petit, and C. R. Cowburn, Science 309, 1688 (2005).

[5] M. Pannetier, C. Fermon, G. L. Goff, J. Simola, and E. Kerr, Science 304, 1648 (2004).

[6] D. Weller and A. Moser, IEEE Trans. Magn. 35, 4423 (1999).

[7] R. H. Koch, G. Grinstein, G. A. Keefe, Y. Lu, P. L. Trouilloud, W. J. Gallagher, and S. S. P. Parkin, Phys. Rev. Lett. 84, 5419 (2000).

[8] C. Wuth, L. Kolbe, and G. Meier, J. Appl. Phys. 114, 103901 (2013).
[9] L. Bocklage, F.-U. Stein, M. Martens, T. Matsuyama, and G. Meier, Appl. Phys. Lett. 103, 092406 (2013).

[10] D. E. Endean, C. T. Weigelt, R. H. Victora, and E. D. Dahlberg, Appl. Phys. Lett. 104, 252408 (2014).

[11] F. Coppinger, J. Genoe, D. K. Maude, U. Gennser, J. C. Portal, K. E. Singer, P. Rutter, T. Taskin, A. R. Peaker, and A. C. Wright, Phys. Rev. Lett. 75, 3513 (1995).

[12] F. Coppinger, J. Genoe, D. K. Maude, X. Kleber, L. B. Rigal, U. Gennser, J. C. Portal, K. E. Singer, P. Rutter, T. Taskin, A. R. Peaker, and A. C. Wright, Phys. Rev. B 57, 7182 (1998).

[13] W. Wernsdorfer, E. B. Orozco, K. Hasselbach, A. Benoit, B. Barbara, N. Demoncy, A. Loiseau, H. Pascard, and D. Mailly, Phys. Rev. Lett. 78, 1791 (1997). 
[14] M. Bode, O. Pietzsch, A. Kubetzka, and R. Wiesendanger, Phys. Rev. Lett. 92, 067201 (2004).

[15] J. A. J. Burgess, A. E. Fraser, F. Fani Sani, D. Vick, B. D. Hauer, J. P. Davis, and M. R. Freeman, Science 339, 1051 (2013).

[16] A. Singh and A. Ghosh, Phys. Rev. B 84, 060407(R) (2011).

[17] L. Jiang, E. R. Nowak, P. E. Scott, J. Johnson, J. M. Slaughter, J. J. Sun, and R. W. Dave, Phys. Rev. B 69, 054407 (2004).

[18] M. Ramsteiner, H. Y. Hao, A. Kawaharazuka, H. J. Zhu, M. Kästner, R. Hey, L. Däweritz, H. T. Grahn, and K. H. Ploog, Phys. Rev. B 66, 081304(R) (2002).

[19] C. Pampuch, A. K. Das, A. Ney, L. Däweritz, R. Koch, and K. H. Ploog, Phys. Rev. Lett. 91, 147203 (2003).

[20] M. T. Elm and S. Hara, Adv. Mater. 26, 8079 (2014).

[21] M. T. Elm, C. Michel, J. Stehr, D. M. Hofmann, P. J. Klar, S. Ito, S. Hara, and H.-A. Krug von Nidda, J. Appl. Phys. 107, 013701 (2010).

[22] M. T. Elm, P. J. Klar, S. Ito, and S. Hara, Phys. Rev. B 83, 235305 (2011).

[23] S. Hara, D. Kawamura, H. Iguchi, J. Motohisa, and T. Fukui, J. Cryst. Growth 310, 2390 (2008).

[24] M. Fischer, M. T. Elm, S. Sakita, S. Hara, and P. J. Klar, Appl. Phys. Lett. 106, 032401 (2015).

[25] S. Ito, S. Hara, T. Wakatsuki, and T. Fukui, Appl. Phys. Lett. 94, 243117 (2009).

[26] T. Wakatsuki, S. Hara, S. Ito, D. Kawamura, and T. Fukui, Jpn. J. Appl. Phys. 48, 04C137 (2009).

[27] K. Komagata, S. Hara, S. Ito, and T. Fukui, Jpn. J. Appl. Phys. 50, $06 \mathrm{GH} 01$ (2011).

[28] H. Kato, S. Sakita, and S. Hara, J. Cryst. Growth 414, 151 (2015).

[29] N. Clément, K. Nishiguchi, A. Fujiwara, and D. Vuillaume, Nat. Commun. 1, 92 (2010).

[30] B. Doudin, G. Redmond, S. E. Gilbert, and J.-Ph. Ansermet, Phys. Rev. Lett. 79, 933 (1997).

[31] S. Ingvarsson, G. Xiao, S. S. P. Parkin, W. J. Gallagher, G. Grinstein, and R. H. Koch, Phys. Rev. Lett. 85, 3289 (2000).

[32] C. Heiliger, M. Czerner, P. J. Klar, and S. Hara, IEEE Trans. Magn. 46, 1702 (2010).

[33] M. T. Elm, P. J. Klar, S. Ito, S. Hara, and H.-A. Krug von Nidda, Phys. Rev. B 84, 035309 (2011).

[34] L. Néel, C. R. Hebd. Seances Acad. Sci. 228, 664 (1949).

[35] T. Hartmann, M. Lampalzer, P. J. Klar, W. Stolz, W. Heimbrodt, H.-A. Krug von Nidda, A. Loidl, and L. Svistov, Phys. E (Amsterdam, Neth.) 13, 572 (2002).
[36] W. F. Brown, Phys. Rev. 130, 1677 (1963).

[37] R. Engel-Herbert and T. Hesjedal, J. Magn. Magn. Mater. 323, 1840 (2011).

[38] H. Akinaga, J. De Boeck, G. Borghs, S. Miyanishi, A. Asamitsu, W. Van Roy, Y. Tomioka, and L. H. Kuo, Appl. Phys. Lett. 72, 3368 (1998).

[39] K. Shimada, O. Rader, A. Fujimori, A. Kimura. K. Ono, N. Kamakura, A. Kakizaki, M. Tanaka, and M. Shirai, J. Electron Spectrosc. Relat. Phenom. 101, 383 (1999).

[40] Gmelin Handbook of Inorganic and Organometallic Chemistry, 8th ed., edited by H. Katscher et al., Vol. 56, C9, Compounds of Manganese with Phosphorus, Arsenic, Antimony (Springer, Berlin, 1983).

[41] C. Kittel, Phys. Rev 73, 155 (1948).

[42] M. T. Elm, Ph.D. thesis, Justus-Liebig University Gießen, 2010.

[43] F. D. Stacey, Proc. Phys. Soc. London 73, 136 (1959).

[44] D. L. Leslie-Pelecki and R. D. Rieke, Chem. Mater. 8, 1770 (1996).

[45] D. P. E. Dickson, N. M. K. Reid, C. Hunt, H. D. Williams, M. El-Hilo, and K. O’Grady, J. Magn. Magn. Mater. 125, 345 (1993).

[46] G. Xiao, S. H. Liou, A. Levy, J. N. Taylor, and C. L. Chien, Phys. Rev. B 34, 7573 (1986)

[47] W. Wernsdorfer, B. Doudin, D. Mailly, K. Hasselbach, A Benoit, J. Meier, J.-Ph. Ansermet, and B. Barbara, Phys. Rev. Lett. 77, 1873 (1996).

[48] I. Klik and L. Gunther, J. Stat. Phys. 60, 473 (1990).

[49] W. Wernsdorfer, K. Hasselbach, D. Mailly, B. Barbara, A. Benoit, L. Thomas, and G. Suran, J. Magn. Magn. Mater 145, 33 (1995).

[50] M. Lederman, S. Schultz, and M. Ozaki, Phys. Rev. Lett. 73 , 1986 (1994).

[51] L. San, Emeterio Alvarez, K.-Y. Wang, S. Lepadatu, S. Landi, S. J. Bending, and C. H. Marrows, Phys. Rev. Lett. 104, 137205 (2010).

[52] Y. Nakatani, A. Thiaville, and J. Miltat, Nat. Mater. 2, 521 (2003).

[53] D.-H. Kim, S.-B. Choe, and S.-C. Shin, Phys. Rev. Lett. 90, 087203 (2003).

[54] M.-Y. Im, L. Bocklage, P. Fischer, and G. Meier, Phys. Rev. Lett. 102, 147204 (2009).

[55] A. Singh, S. Mukhopadhyay, and A. Ghosh, Phys. Rev. Lett. 105, 067206 (2010). 